\title{
Search for a Higgs boson decaying into two photons in $\mathrm{e}^{+} \mathrm{e}^{-}$interactions at $\sqrt{s}=189 \mathrm{GeV}$
}

\section{L3 Collaboration}

M. Acciarri ${ }^{\mathrm{z}}$, P. Achard ${ }^{\mathrm{s}}$, O. Adriani ${ }^{\mathrm{p}}, \mathrm{M}$. Aguilar-Benitez ${ }^{\mathrm{y}}, \mathrm{J}$. Alcaraz $^{\mathrm{y}}$, G. Alemanni ${ }^{\mathrm{v}}$, J. Allaby ${ }^{\mathrm{q}}$, A. Aloisio ${ }^{\mathrm{ab}}$, M.G. Alviggi ${ }^{\mathrm{ab}}$, G. Ambrosi ${ }^{\mathrm{s}}$, H. Anderhub av , V.P. Andreev f,aj, T. Angelescu ${ }^{1}$, F. Anselmo ${ }^{\mathrm{i}}$, A. Arefiev ${ }^{\text {aa }}$, T. Azemoon ${ }^{\text {c }}$, T. Aziz ${ }^{\text {j }}$, P. Bagnaia ${ }^{\text {ai }}$, A. Bajo ${ }^{\mathrm{y}}$, L. Baksay $^{\mathrm{aq}}$, A. Balandras ${ }^{\mathrm{d}}$, S.V. Baldew ${ }^{\text {b }, ~ S . ~ B a n e r j e e ~}{ }^{j}$, Sw. Banerjee ${ }^{j}$, A. Barczyk ${ }^{\text {av,at }}$, R. Barillère ${ }^{q}$, P. Bartalini ${ }^{\mathrm{v}}$, M. Basile ${ }^{\mathrm{i}}$, R. Battiston ${ }^{\text {af }}$, A. Bay ${ }^{\mathrm{v}}$, F. Becattini $^{\mathrm{p}}, \mathrm{U}^{\mathrm{B}}$ Becker $^{\mathrm{n}}$, F. Behner ${ }^{\text {av }}$, L. Bellucci ${ }^{\mathrm{p}}$, R. Berbeco ${ }^{\mathrm{c}}$, J. Berdugo ${ }^{\mathrm{y}}$, P. Berges ${ }^{\mathrm{n}}$, B. Bertucci ${ }^{\text {af }}$, B.L. Betev ${ }^{\text {av }}$, S. Bhattacharya ${ }^{j}$, M. Biasini ${ }^{\text {af }}$, A. Biland ${ }^{\text {av }}$, J.J. Blaising ${ }^{\mathrm{d}}$, S.C. Blyth ${ }^{\mathrm{ag}}$, G.J. Bobbink ${ }^{\mathrm{b}}$, A. Böhm ${ }^{\mathrm{a}}$, L. Boldizsar ${ }^{\mathrm{m}}$, B. Borgia ${ }^{\text {ai }}$, D. Bourilkov ${ }^{\mathrm{av}}$, M. Bourquin ${ }^{\text {s }, ~ S . ~ B r a c c i n i ~}{ }^{\text {s, J.G. Branson }}{ }^{\text {am }}$, F. Brochu ${ }^{\text {d }}$, A. Buffini ${ }^{p}$, A. Buijs ${ }^{\text {ar }}$, J.D. Burger ${ }^{\text {n, W.J. Burger }}{ }^{\text {af }}$, X.D. Cai ${ }^{\text {n }}$, M. Capell ${ }^{\text {n }}$, G. Cara Romeo ${ }^{\text {i }}$, G. Carlino $^{\mathrm{ab}}$, A.M. Cartacci ${ }^{\mathrm{p}}$, J. Casaus ${ }^{\mathrm{y}}$, G. Castellini ${ }^{\mathrm{p}}$, F. Cavallari ${ }^{\text {ai }}$, N. Cavallo ${ }^{\text {ak }}$, C. Cecchi ${ }^{\text {af }}$, M. Cerrada ${ }^{y}$, F. Cesaroni ${ }^{\text {w }}$, M. Chamizo ${ }^{\text {s }}$, Y.H. Chang ${ }^{\text {ax }}$, U.K. Chaturvedi $^{\mathrm{r}}$, M. Chemarin ${ }^{\mathrm{x}}$, A. Chen ${ }^{\mathrm{ax}}$, G. Chen ${ }^{\mathrm{g}}$, G.M. Chen ${ }^{\mathrm{g}}$, H.F. Chen ${ }^{\mathrm{t}}$, H.S. Chen ${ }^{\mathrm{g}}$, G. Chiefari ${ }^{\mathrm{ab}}$, L. Cifarelli ${ }^{\text {al }}$, F. Cindolo ${ }^{\mathrm{i}}$, C. Civinini ${ }^{\mathrm{p}}{ }^{\text {, I. Clare }}{ }^{\mathrm{n}}$, R. Clare ${ }^{\mathrm{n}}$, G. Coignet ${ }^{\mathrm{d}}, \mathrm{N}$. Colino ${ }^{\mathrm{y}}, \mathrm{S}$. Costantini ${ }^{\mathrm{e}}, \mathrm{F}$. Cotorobai ${ }^{1}$, B. de la Cruz ${ }^{\mathrm{y}}$, A. Csilling ${ }^{\mathrm{m}}$, S. Cucciarelli ${ }^{\text {af }}$, T.S. Dai ${ }^{\mathrm{n}}$, J.A. van Dalen ${ }^{\text {ad }}$, R. D'Alessandro ${ }^{\mathrm{p}}$, R. de Asmundis ${ }^{\mathrm{ab}}$, P. Déglon ${ }^{\mathrm{s}}$, A. Degré ${ }^{\mathrm{d}}$, K. Deiters ${ }^{\text {at }}$, D. della Volpe ${ }^{\mathrm{ab}}$, E. Delmeire ${ }^{\mathrm{s}}$, P. Denes ${ }^{\text {ah }}$, F. DeNotaristefani ${ }^{\text {ai }}$, A. De Salvo ${ }^{\text {av }}$, M. Diemoz ${ }^{\text {ai }}$, M. Dierckxsens ${ }^{\mathrm{b}}$, D. van Dierendonck ${ }^{\mathrm{b}}$, C. Dionisi ${ }^{\text {ai }}$, M. Dittmar ${ }^{\text {av }}$, A. Dominguez ${ }^{\mathrm{am}}$, A. Doria $^{\mathrm{ab}}$, M.T. Dova ${ }^{\mathrm{r}, 5}$, D. Duchesneau ${ }^{\mathrm{d}}$, D. Dufournaud ${ }^{\mathrm{d}}$, P. Duinker ${ }^{\mathrm{b}}$, I. Duran ${ }^{\text {an }}$, H. El Mamouni ${ }^{\mathrm{x}}$, A. Engler ${ }^{\mathrm{ag}}$, F.J. Eppling ${ }^{\text {n }}$, F.C. Erné $^{\mathrm{b}}$, P. Extermann ${ }^{\mathrm{s}}$, M. Fabre ${ }^{\text {at }}$, M.A. Falagan ${ }^{\mathrm{y}}$, S. Falciano ${ }^{\text {ai, }}$, A. Favara ${ }^{\mathrm{q}}$, J. Fay ${ }^{\mathrm{x}}$, O. Fedin ${ }^{\text {aj }}$, M. Felcini ${ }^{\text {av }}$, T. Ferguson $^{\text {ag }}$, H. Fesefeldt ${ }^{\text {a }}$, E. Fiandrini ${ }^{\text {af }}$, J.H. Field ${ }^{\text {s }}$, F. Filthaut ${ }^{\mathrm{q}}$, P.H. Fisher ${ }^{n}$, I. Fisk ${ }^{\text {am }}$, G. Forconi ${ }^{n}$, K. Freudenreich ${ }^{\text {av }}$, C. Furetta ${ }^{\text {, }}$, Yu. Galaktionov aa,n, S.N. Ganguli ${ }^{\text {j, }}$ P. Garcia-Abia ${ }^{\mathrm{e}}$, M. Gataullin ${ }^{\text {ae }}$, S.S. Gau ${ }^{\mathrm{k}}$, 
S. Gentile ${ }^{\text {ai, }}$, N. Gheordanescu ${ }^{1}$, S. Giagu ${ }^{\text {ai }}$, Z.F. Gong ${ }^{\text {t }}$, G. Grenier ${ }^{x}$, O. Grimm ${ }^{\text {av }}$, M.W. Gruenewald ${ }^{\text {h }}$, M. Guida ${ }^{\text {al }}$, R. van Gulik ${ }^{\text {b }}$, V.K. Gupta ${ }^{\text {ah }}$, A. Gurtu ${ }^{\text {j }}$, L.J. Gutay ${ }^{\text {as }}$, D. Haas ${ }^{\text {e }}$, A. Hasan ${ }^{\text {ac }}$, D. Hatzifotiadou ${ }^{\text {i }}$, T. Hebbeker ${ }^{\text {h }}$, A. Hervé ${ }^{\text {, }}$, P. Hidas ${ }^{\text {m}}$, J. Hirschfelder ${ }^{\text {ag }}$, H. Hofer ${ }^{\text {av }}$, G. Holzner ${ }^{\text {av }}$, H. Hoorani ${ }^{\text {ag }}$, S.R. Hou ${ }^{\text {ax }}$, Y. Hu ${ }^{\text {ad }}$, I. Iashvili ${ }^{\text {au }}$, B.N. Jin ${ }^{g}$, L.W. Jones ${ }^{c}$, P. de Jong ${ }^{\text {b }}$, I. Josa-Mutuberría ${ }^{\mathrm{y}}$, R.A. Khan $^{\text {r }}$, M. Kaur ${ }^{\text {r,6 }}$, M.N. Kienzle-Focacci ${ }^{\text {s }}$, D. Kim ${ }^{\text {ai }}$, J.K. Kim ${ }^{\text {ap }}$, J. Kirkby ${ }^{\text {q }}$, D. Kiss ${ }^{\text {m }}$, W. Kittel ${ }^{\text {ad }}$, A. Klimentov ${ }^{\text {n,aa }}$, A.C. König ${ }^{\text {ad }}$, A. Kopp ${ }^{\text {au }}$, V. Koutsenko ${ }^{\text {naa }}$, M. Kräber ${ }^{\text {av }}$, R.W. Kraemer ${ }^{\text {ag }}$, W. Krenz ${ }^{\text {a }}$, A. Krüger ${ }^{\text {au }}$, A. Kunin ${ }^{\text {naa }}$, P. Ladron de Guevara ${ }^{\mathrm{y}}$, I. Laktineh ${ }^{\mathrm{x}}$, G. Landi ${ }^{\mathrm{p}}$, M. Lebeau ${ }^{\mathrm{q}}$, A. Lebedev ${ }^{n}$, P. Lebrun ${ }^{x}$, P. Lecomte ${ }^{\text {av }}$, P. Lecoq ${ }^{q}$, P. Le Coultre ${ }^{\text {av }}$, H.J. Lee ${ }^{\text {h }}$, J.M. Le Goff ${ }^{\mathrm{q}}$, R. Leiste ${ }^{\text {au }}$, P. Levtchenko ${ }^{\text {aj }}$, C. Li $^{\text {t }}$, S. Likhoded ${ }^{\text {au }}$, C.H. Lin ${ }^{\text {ax }}$, W.T. Lin ${ }^{a x}$, F.L. Linde ${ }^{b}$, L. Lista ${ }^{\text {ab }}$, Z.A. Liu ${ }^{\mathrm{g}}$, W. Lohmann ${ }^{\mathrm{au}}$, E. Longo ${ }^{\mathrm{a}}$, Y.S. Lu ${ }^{g}$, K. Lübelsmeyer ${ }^{\text {a }}$, C. Luci ${ }^{\text {q,ai }}$, D. Luckey ${ }^{n}$, L. Lugnier ${ }^{\mathrm{x}}$, L. Luminari ${ }^{\text {ai }}$, W. Lustermann ${ }^{\text {av }}$, W.G. Ma $^{\mathrm{t}}$, M. Maity ${ }^{\mathrm{j}}$, L. Malgeri ${ }^{\mathrm{q}}$, A. Malinin ${ }^{\mathrm{q}}$, C. Maña ${ }^{\mathrm{y}}$, D. Mangeol ${ }^{\text {ad }}$, J. Mans ${ }^{\text {ah }}$, G. Marian ${ }^{\mathrm{o}}$, J.P. Martin ${ }^{\mathrm{x}}$, F. Marzano ai , K. Mazumdar ${ }^{j}$, R.R. McNeil ${ }^{f}$, S. Mele ${ }^{\mathrm{q}}$, L. Merola ${ }^{\text {ab }}$, M. Meschini ${ }^{\mathrm{p}}$, W.J. Metzger ad, M. von der Mey ${ }^{a}$, A. Mihul ${ }^{1}$, H. Milcent ${ }^{\mathrm{q}}$, G. Mirabelli ai, J. Mnich ${ }^{\mathrm{q}}$, G.B. Mohanty ${ }^{\mathrm{j}}$, T. Moulik ${ }^{\mathrm{j}}$, G.S. Muanza ${ }^{\mathrm{x}}$, A.J.M. Muijs ${ }^{\mathrm{b}}$, B. Musicar ${ }^{\text {am }}$, M. Musy ${ }^{\text {ai }}$, M. Napolitano ${ }^{\text {ab }}$, F. Nessi-Tedaldi ${ }^{\text {av }}$, H. Newman ${ }^{\text {ae }}$, T. Niessen ${ }^{\text {a }}$, A. Nisati ${ }^{\text {ai }}$, H. Nowak ${ }^{\text {au }}$, R. Ofierzynski ${ }^{\text {av }}$, G. Organtini ${ }^{\text {ai }}$, A. Oulianov ${ }^{\text {aa }}$, C. Palomares ${ }^{\mathrm{y}}$, D. Pandoulas ${ }^{\text {a }}$, S. Paoletti ${ }^{\text {ai, }}$, P. Paolucci ${ }^{\text {ab }}$, R. Paramatti ai ${ }^{\text {, H.K. Park }}{ }^{\text {ag }}$, I.H. Park ${ }^{\text {ap }}$, G. Passaleva ${ }^{\text {q, }}$, S. Patricelli ${ }^{\text {ab }}$, T. Paul ${ }^{\text {k }}$, M. Pauluzzi ${ }^{\text {af }}$, C. Paus ${ }^{\mathrm{q}}$, F. Pauss ${ }^{\text {av }}$, M. Pedace ${ }^{\text {ai }}$, S. Pensotti ${ }^{z}$, D. Perret-Gallix ${ }^{d}$, B. Petersen ${ }^{\text {ad }}$, D. Piccolo $^{\text {ab }}$, F. Pierella ${ }^{i}$, M. Pieri ${ }^{\text {p }}$, P.A. Piroué ${ }^{\text {ah }}$, E. Pistolesi ${ }^{z}$, V. Plyaskin ${ }^{\text {aa }}$, M. Pohl ${ }^{\text {s, }}$ V. Pojidaev ${ }^{\text {aa, }}$, H. Postema ${ }^{n}$, J. Pothier ${ }^{q}$, D.O. Prokofiev ${ }^{\text {as }}$, D. Prokofiev aj, J. Quartieri ${ }^{\text {al }}$, G. Rahal-Callot ${ }^{\text {av, }}{ }^{\text {, }}$, M.A. Rahaman ${ }^{j}$, P. Raics ${ }^{\circ}$, N. Raja ${ }^{\mathrm{j}}$, R. Ramelli ${ }^{\text {av }}$, P.G. Rancoita ${ }^{\mathrm{z}}$, R. Ranieri ${ }^{\mathrm{p}}$, A. Raspereza ${ }^{\mathrm{au}}$, G. Raven $^{\mathrm{am}}{ }^{\text {, }}$ P. Razis ${ }^{\text {ac }}$, D. Ren ${ }^{\text {av }}$, M. Rescigno ${ }^{\text {ai }}$, S. Reucroft ${ }^{k}$, S. Riemann ${ }^{\text {au }}$, K. Riles ${ }^{c}$, J. Rodin ${ }^{\mathrm{aq}}$, B.P. Roe ${ }^{\mathrm{c}}$, L. Romero ${ }^{\mathrm{y}}$, A. Rosca ${ }^{\mathrm{h}}$, S. Rosier-Lees ${ }^{\mathrm{d}}$, J.A. Rubio ${ }^{\mathrm{q}}$, G. Ruggiero ${ }^{\text {p }}$, H. Rykaczewski ${ }^{\text {av }}$, S. Saremi ${ }^{\text {f }}$, S. Sarkar ${ }^{\text {ai }}$, J. Salicio ${ }^{\text {q }}$, E. Sanchez ${ }^{\text {q }}$, M.P. Sanders ${ }^{\text {ad }}$, M.E. Sarakinos ${ }^{\text {u }}$, C. Schäfer ${ }^{\text {q }}$, V. Schegelsky ${ }^{\text {aj }}$, S. Schmidt-Kaerst ${ }^{\text {a }}$, D. Schmitz ${ }^{\text {a }}$, H. Schopper ${ }^{\text {aw }}$, D.J. Schotanus ${ }^{\text {ad }}$, G. Schwering ${ }^{\text {a }}$, C. Sciacca ${ }^{\text {ab }}$, A. Seganti ${ }^{\text {i }}$, L. Servoli ${ }^{\text {af }}$, S. Shevchenko ${ }^{\text {ae }}$, N. Shivarov ${ }^{\text {ao }}$, V. Shoutko aa, E. Shumilov ${ }^{\text {aa }}$, A. Shvorob ${ }^{\text {ae }}$, T. Siedenburg ${ }^{\text {a }}$, D. Son ${ }^{\text {ap }}$, B. Smith ${ }^{\text {ag }}$, P. Spillantini ${ }^{p}$, M. Steuer ${ }^{n}$, D.P. Stickland ${ }^{\text {ah }}$, A. Stone ${ }^{f}$, B. Stoyanov ${ }^{\text {ao }}$, A. Straessner ${ }^{\text {a }}$, K. Sudhakar ${ }^{\mathrm{j}}$, G. Sultanov ${ }^{\mathrm{r}}$, L.Z. Sun ${ }^{\mathrm{t}}$, H. Suter ${ }^{\text {av }}$, J.D. Swain ${ }^{\mathrm{r}}$, Z. Szillasi ${ }^{\text {aq,3, }}$, T. Sztaricskai aq,3 X.W. Tang ${ }^{\mathrm{g}}$, L. Tauscher ${ }^{\mathrm{e}}$, L. Taylor ${ }^{\mathrm{k}}$, B. Tellili ${ }^{\mathrm{x}}$, 
C. Timmermans ${ }^{\text {ad }}$, Samuel C.C. Ting ${ }^{n}$, S.M. Ting ${ }^{n}$, S.C. Tonwar ${ }^{\mathrm{j}}$, J. Tóth ${ }^{\mathrm{m}}$, C. Tully ${ }^{\mathrm{q}}$, K.L. Tung ${ }^{\mathrm{g}}$, Y. Uchida ${ }^{\mathrm{n}}$, J. Ulbricht ${ }^{\text {av }}$, E. Valente ${ }^{\text {ai }}$, G. Vesztergombi ${ }^{\mathrm{m}}$, I. Vetlitsky ${ }^{\text {aa }}$, D. Vicinanza ${ }^{\text {al }}$, G. Viertel ${ }^{\text {av }}$, S. Villa ${ }^{k}$, M. Vivargent ${ }^{d}$, S. Vlachos ${ }^{e}$, I. Vodopianov ${ }^{\text {aj }}$, H. Vogel ${ }^{\text {ag }}$, H. Vogt ${ }^{\text {au }}$, I. Vorobiev ${ }^{\text {aa }}$, A.A. Vorobyov ${ }^{\text {aj, }}$ A. Vorvolakos ${ }^{\text {ac }}$, M. Wadhwa ${ }^{\mathrm{e}}$, W. Wallraff ${ }^{\mathrm{a}}$, M. Wang ${ }^{\mathrm{n}}$, X.L. Wang ${ }^{\mathrm{t}}$, Z.M. Wang ${ }^{\mathrm{t}}$, A. Weber ${ }^{\mathrm{a}}$, M. Weber ${ }^{\mathrm{a}}$, P. Wienemann ${ }^{\mathrm{a}}$, H. Wilkens ${ }^{\text {ad }}$, S.X. Wu ${ }^{\text {n, }}$ S. Wynhoff ${ }^{\mathrm{q}}$, L. Xia $^{\mathrm{ae}}$, Z.Z. Xu ${ }^{\mathrm{t}}$, J. Yamamoto ${ }^{\mathrm{c}}$, B.Z. Yang ${ }^{\mathrm{t}}$, C.G. Yang ${ }^{\mathrm{g}}$, H.J. Yang ${ }^{\mathrm{g}}$, M. Yang ${ }^{\mathrm{g}}$, J.B. Ye ${ }^{\mathrm{t}}$, S.C. Yeh ${ }^{\text {ay }}$, An. Zalite ${ }^{\text {aj }}$, Yu. Zalite ${ }^{\text {aj }}$, Z.P. Zhang ${ }^{\text {t }}$, G.Y. Zhu ${ }^{\text {g }}$, R.Y. Zhu ${ }^{\text {ae }}$, A. Zichichi ${ }^{\text {i,q,r }}$, G. Zilizi aq,3, B. Zimmermann ${ }^{\text {av }}$, M. Zöller ${ }^{\text {a }}$

\footnotetext{
${ }^{a}$ I. Physikalisches Institut, RWTH, D-52056 Aachen, Germany,

and III. Physikalisches Institut, RWTH, D-52056 Aachen, Germany ${ }^{I}$

${ }^{\mathrm{b}}$ National Institute for High Energy Physics, NIKHEF, and University of Amsterdam, NL-1009 DB Amsterdam, The Netherlands

${ }^{\mathrm{c}}$ University of Michigan, Ann Arbor, MI 48109, USA

' Laboratoire d'Annecy-le-Vieux de Physique des Particules, LAPP, IN2P3-CNRS, BP 110, F-74941 Annecy-le-Vieux CEDEX, France

${ }^{\mathrm{e}}$ Institute of Physics, University of Basel, CH-4056 Basel, Switzerland

${ }^{\mathrm{f}}$ Louisiana State University, Baton Rouge, LA 70803, USA

g Institute of High Energy Physics, IHEP, 100039 Beijing, China ${ }^{7}$

${ }^{\mathrm{h}}$ Humboldt University, D-10099 Berlin, Germany

${ }^{\mathrm{i}}$ University of Bologna and INFN-Sezione di Bologna, I-40126 Bologna, Italy

${ }^{\mathrm{j}}$ Tata Institute of Fundamental Research, Bombay 400 005, India

${ }^{\mathrm{k}}$ Northeastern University, Boston, MA 02115, USA

${ }^{1}$ Institute of Atomic Physics and University of Bucharest, R-76900 Bucharest, Romania

${ }^{m}$ Central Research Institute for Physics of the Hungarian Academy of Sciences, H-1525 Budapest 114, Hungary ${ }^{2}$

${ }^{\mathrm{n}}$ Massachusetts Institute of Technology, Cambridge, MA 02139, USA

${ }^{\circ}$ KLTE-ATOMKI, H-4010 Debrecen, Hungary ${ }^{3}$

${ }^{\mathrm{p}}$ INFN Sezione di Firenze and University of Florence, I-50125 Florence, Italy

${ }^{\mathrm{q}}$ European Laboratory for Particle Physics, CERN, CH-1211 Geneva 23, Switzerland

${ }^{\mathrm{r}}$ World Laboratory, FBLJA Project, CH-1211 Geneva 23, Switzerland

${ }^{\mathrm{s}}$ University of Geneva, CH-1211 Geneva 4, Switzerland

${ }^{\mathrm{t}}$ Chinese University of Science and Technology, USTC, Hefei, Anhui 230 029, China ${ }^{7}$

u SEFT, Research Institute for High Energy Physics, P.O. Box 9, SF-00014 Helsinki, Finland

${ }^{v}$ University of Lausanne, CH-1015 Lausanne, Switzerland

${ }^{\mathrm{w}}$ INFN-Sezione di Lecce and Universitá Degli Studi di Lecce, I-73100 Lecce, Italy

${ }^{x}$ Institut de Physique Nucléaire de Lyon, IN2P3-CNRS, Université Claude Bernard, F-69622 Villeurbanne, France

${ }^{y}$ Centro de Investigaciones Energéticas, Medioambientales y Tecnologícas, CIEMAT, E-28040 Madrid, Spain ${ }^{4}$

${ }^{\mathrm{z}}$ INFN-Sezione di Milano, I-20133 Milan, Italy

aa Institute of Theoretical and Experimental Physics, ITEP, Moscow, Russia

${ }^{\mathrm{ab}}$ INFN-Sezione di Napoli and University of Naples, I-80125 Naples, Italy

ac Department of Natural Sciences, University of Cyprus, Nicosia, Cyprus

${ }^{\text {ad }}$ University of Nijmegen and NIKHEF, NL-6525 ED Nijmegen, The Netherlands

${ }^{\text {ae }}$ California Institute of Technology, Pasadena, CA 91125, USA

af INFN-Sezione di Perugia and Universitá Degli Studi di Perugia, I-06100 Perugia, Italy

ag Carnegie Mellon University, Pittsburgh, PA 15213, USA

${ }^{\text {ah }}$ Princeton University, Princeton, NJ 08544, USA

ai INFN-Sezione di Roma and University of Rome, "La Sapienza", I-00185 Rome, Italy

${ }^{\text {aj }}$ Nuclear Physics Institute, St. Petersburg, Russia

${ }^{\mathrm{ak}}$ INFN-Sezione di Napoli and University of Potenza, I-85100 Potenza, Italy

${ }^{\text {al }}$ University and INFN, Salerno, I-84100 Salerno, Italy

am University of California, San Diego, CA 92093, USA

${ }^{a n}$ Dept. de Fisica de Particulas Elementales, Univ. de Santiago, E-15706 Santiago de Compostela, Spain

ao Bulgarian Academy of Sciences, Central Lab. of Mechatronics and Instrumentation, BU-1113 Sofia, Bulgaria
} 
ap Laboratory of High Energy Physics, Kyungpook National University, 702-701 Taegu, South Korea

aq University of Alabama, Tuscaloosa, AL 35486, USA

${ }^{\text {ar }}$ Utrecht University and NIKHEF, NL-3584 CB Utrecht, The Netherlands

as Purdue University, West Lafayette, IN 47907, USA

${ }^{\text {at }}$ Paul Scherrer Institut, PSI, CH-5232 Villigen, Switzerland

au DESY, D-15738 Zeuthen, Germany

${ }^{\text {av }}$ Eidgenössische Technische Hochschule, ETH Zürich, CH-8093 Zürich, Switzerland

aw University of Hamburg, D-22761 Hamburg, Germany

${ }^{a x}$ National Central University, Chung-Li, Taiwan, ROC

ay Department of Physics, National Tsing Hua University, Taiwan, ROC

Received 24 July 2000; accepted 8 August 2000

Editor: K. Winter

\begin{abstract}
A search is performed for a Higgs boson produced in association with a $\mathrm{Z}$ boson and decaying into two photons, using the $\mathrm{L} 3$ data collected at LEP at a centre-of-mass energy of $189 \mathrm{GeV}$. All decay modes of the $\mathrm{Z}$ are considered. No signal is observed and limits on the branching fraction of the Higgs boson decay into two photons as a function of the Higgs mass are derived assuming a Standard Model production rate. A lower limit on the mass of a fermiophobic Higgs is set at $94.9 \mathrm{GeV}$ at 95\% confidence level. (C) 2000 Elsevier Science B.V. All rights reserved.
\end{abstract}

\section{Introduction}

In the Standard Model, the decay of a Higgs boson $\mathrm{h}$ into a photon pair occurs at the one loop level and its branching fraction is small [1]. For example, for Higgs masses in the range $80<M_{\mathrm{h}}<$ $110 \mathrm{GeV}$, this decay rate lies between $0.1 \%$ and $0.2 \%$. However, several extended models predict enhancements of this branching fraction [2]. In Two Higgs Doublet Models of Type I [3], with an appropriate choice of the model parameters, the lightest

\footnotetext{
E-mail address: Salvatore.Mele@cern.ch (S. Mele).

${ }^{1}$ Supported by the German Bundesministerium für Bildung, Wissenschaft, Forschung und Technologie.

${ }^{2}$ Supported by the Hungarian OTKA fund under contract numbers T019181, F023259 and T024011.

${ }^{3}$ Also supported by the Hungarian OTKA fund under contract numbers T22238 and T026178.

${ }^{4}$ Supported also by the Comisión Interministerial de Ciencia y Tecnología.

${ }^{5}$ Also supported by CONICET and Universidad Nacional de La Plata, CC 67, 1900 La Plata, Argentina.

${ }^{6}$ Also supported by Panjab University, Chandigarh-160014, India.

${ }^{7}$ Supported by the National Natural Science Foundation of China.
}

CP even Higgs boson does not couple to fermions at tree level. Such a Higgs is expected to decay dominantly into a pair of photons if its mass is below $90 \mathrm{GeV}$ [4].

We search for a Higgs boson produced in association with a $\mathrm{Z}$ boson through the process $\mathrm{e}^{+} \mathrm{e}^{-} \rightarrow \mathrm{Zh}$, followed by the decay $\mathrm{h} \rightarrow \gamma \gamma$, in all decay modes of the $\mathrm{Z}$ boson.

These results supersede previous limits obtained by the L3 Collaboration from data at lower centreof-mass energies [5]. Similar analyses were published by the OPAL [6] and the DELPHI [7] Collaborations.

\section{Data and Monte Carlo samples}

The data were collected with the L3 detector [8] at a centre-of-mass energy $\sqrt{s}=189 \mathrm{GeV}$ and amount to an integrated luminosity of $176.4 \mathrm{pb}^{-1}$.

The Standard Model Higgs production cross section is calculated using the HZHA generator [9]. Monte Carlo samples were generated using PYTHIA [10] for Higgs masses between 50 and $100 \mathrm{GeV}$. For background studies the following Monte Carlo pro- 
grams were used: KK2f [11] $\left(\mathrm{e}^{+} \mathrm{e}^{-} \rightarrow \mathrm{q} \overline{\mathrm{q}}(\gamma)\right)$, PYTHIA $\left(\mathrm{e}^{+} \mathrm{e}^{-} \rightarrow \mathrm{ZZ}\right.$ and $\left.\mathrm{e}^{+} \mathrm{e}^{-} \rightarrow \mathrm{Ze}^{+} \mathrm{e}^{-}\right)$, KORALW [12] $\left(\mathrm{e}^{+} \mathrm{e}^{-} \rightarrow \mathrm{W}^{+} \mathrm{W}^{-}\right)$, PHOJET [13] $\left(\mathrm{e}^{+} \mathrm{e}^{-} \rightarrow\right.$ $\left.\mathrm{e}^{+} \mathrm{e}^{-} \mathrm{q} \overline{\mathrm{q}}\right)$, KORALZ [14] $\left(\mathrm{e}^{+} \mathrm{e}^{-} \rightarrow \nu \bar{\nu}(\gamma)\right)$, GGG [15] $\left(\mathrm{e}^{+} \mathrm{e}^{-} \rightarrow \gamma \gamma(\gamma)\right)$, BHWIDE [16] $\left(\mathrm{e}^{+} \mathrm{e}^{-} \rightarrow \mathrm{e}^{+} \mathrm{e}^{-}(\gamma)\right)$, TEEGG [17] $\left(\mathrm{e}^{+} \mathrm{e}^{-} \rightarrow \mathrm{e}^{+} \mathrm{e}^{-} \gamma(\gamma)\right)$, DIAG36 [18] $\left(\mathrm{e}^{+} \mathrm{e}^{-} \rightarrow \mathrm{e}^{+} \mathrm{e}^{-} \mathrm{e}^{+} \mathrm{e}^{-}\right)$and EXCALIBUR [19] $\left(\mathrm{e}^{+} \mathrm{e}^{-}\right.$ $\left.\rightarrow \mathrm{e}^{+} \mathrm{e}^{-} \nu \bar{\nu}\right)$. The number of simulated events for the most important background channels is at least 100 times higher than the number of expected data events, while this factor is 200 for the expected signal.

The L3 detector response is simulated using the GEANT 3.15 program [20], which takes into account the effects of energy loss, multiple scattering and showers in the detector. GHEISHA [21] is used to simulate hadronic interactions in the detector. Time dependent inefficiencies, as monitored during the data taking, are also simulated.

\section{Analysis procedures}

A cut-based selection is performed in order to select events with photons and to identify the $\mathrm{Z}$ in its various decay modes. This gives rise to $\mathrm{q} \overline{\mathrm{q}} \gamma \gamma, \nu \bar{\nu} \gamma \gamma$ and $\ell^{+} \ell^{-} \gamma \gamma$, with $\ell=\mathrm{e}, \mu, \tau$, final states. The selection criteria for each final state are described in the following sections and rely on a common photon identification.

Photons are identified as clusters in the electromagnetic calorimeter (BGO) with an energy greater than $1 \mathrm{GeV}$ and a shower shape compatible with that of an electromagnetic shower. The ratio of energies deposited in a $3 \times 3$ crystal matrix and a $5 \times 5$ matrix, centred on the shower axis, must be greater than 0.95 . The energy deposition in the hadron calorimeter must not exceed $20 \%$ of the energy deposited in the electromagnetic calorimeter.

In addition, the clusters must not be associated with a charged track within $50 \mathrm{mrad}$ in the plane perpendicular to the beam axis. To suppress photons from initial state radiation, only photons in the polar angle range $45^{\circ}<\theta<135^{\circ}, 25^{\circ}<\theta<35^{\circ}$ or $145^{\circ}<$ $\theta<155^{\circ}$ are accepted, which corresponds to the barrel and end-cap regions of the BGO.

In the following selections we require at least two photons. To ensure that the pair of photons arise from the decay of a heavy resonance we require the energy of the most energetic photon to be larger than $10 \mathrm{GeV}$ and the energy of the second most energetic photon to be larger than $6 \mathrm{GeV}$.

The angular distribution of the di-photon system with respect to the beam direction is flat for photons coming from the Higgs decay, while it peaks at low polar angles for those photons originating from double initial state radiation. Therefore, we require the absolute value of the cosine of the polar angle of the di-photon system not to exceed 0.966 .

\subsection{The $q \bar{q} \gamma \gamma$ final state}

The signature for the $\mathrm{q} \overline{\mathrm{q}} \gamma \gamma$ final state is a pair of isolated photons accompanied by two jets. To select these events, we first apply a hadronic preselection requiring high multiplicity events. The visible energy normalised to the centre-of-mass energy is required to be larger than 0.5 and the energy imbalances parallel and perpendicular to the beam direction, normalised to the visible energy, are required to be below 0.4 . In order to reduce the background from the photon-photon interaction events, we require the energy in a $30^{\circ}$ cone around the beam pipe to be less than half of the visible energy. The yield of this preselection is reported in Table 1.

From this sample we select those events which contain at least two photons. All other particles are clustered in two jets using the DURHAM jet algorithm [22]. To reject photons coming from neutral hadron decays we require them to be isolated: the energy in a $10^{\circ}$ cone around the photon direction must be less than $1.5 \mathrm{GeV}$, and in a $20^{\circ}$ cone less than $3.5 \mathrm{GeV}$. The number of charged tracks and calorimeter clusters in a $20^{\circ}$ cone around the photon direction must be below four. The opening angle between the photons must be larger than $50^{\circ}$ and the angle between the photon direction and the nearest jet must exceed $25^{\circ}$.

Table 1

Number of events expected from Standard Model processes compared to the observed number of events, after the preselection and selection steps, for the $q \bar{q} \gamma \gamma$ final state

\begin{tabular}{ccccccc}
\hline & Data & $\Sigma$ Bkg. & $\mathrm{q} \overline{\mathrm{q}}(\gamma)$ & $\mathrm{WW}$ & $\mathrm{Ze}^{+} \mathrm{e}^{-}$ & $\mathrm{ZZ}$ \\
\hline preselection & 8146 & 8221.7 & 5745.9 & 2309.8 & 58.2 & 109.8 \\
selection & 10 & 16.2 & 16.0 & 0.0 & 0.1 & 0.1 \\
\hline
\end{tabular}



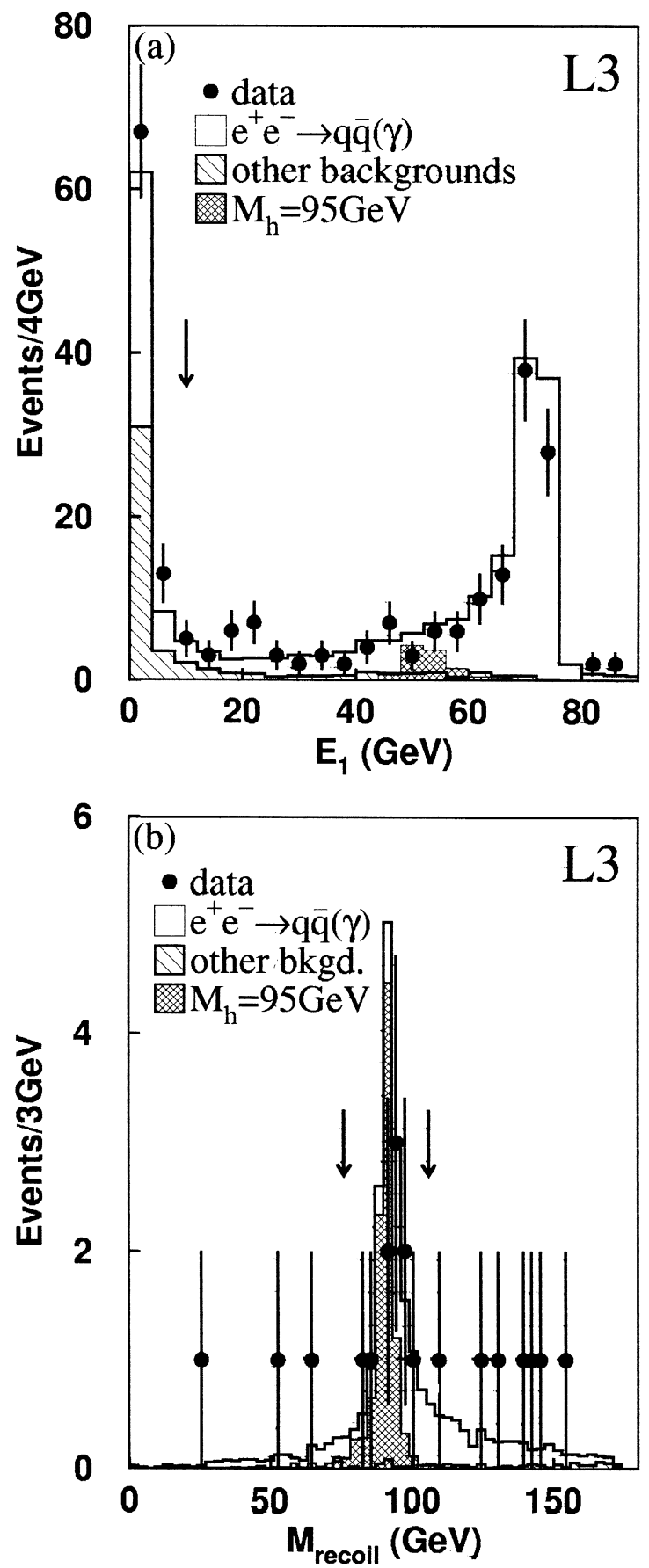

Fig. 1. Distributions for the $q \bar{q} \gamma \gamma$ final state of (a) the energy $E_{1}$ of the most energetic photon and (b) the recoil mass against the di-photon system in data, background and for a Higgs boson signal with the mass $M_{\mathrm{h}}=95 \mathrm{GeV}$. The signal, corresponding to the Standard Model cross section and a $\operatorname{BR}(\mathrm{h} \rightarrow \gamma \gamma)=1$, is superimposed and normalised to the integrated luminosity.
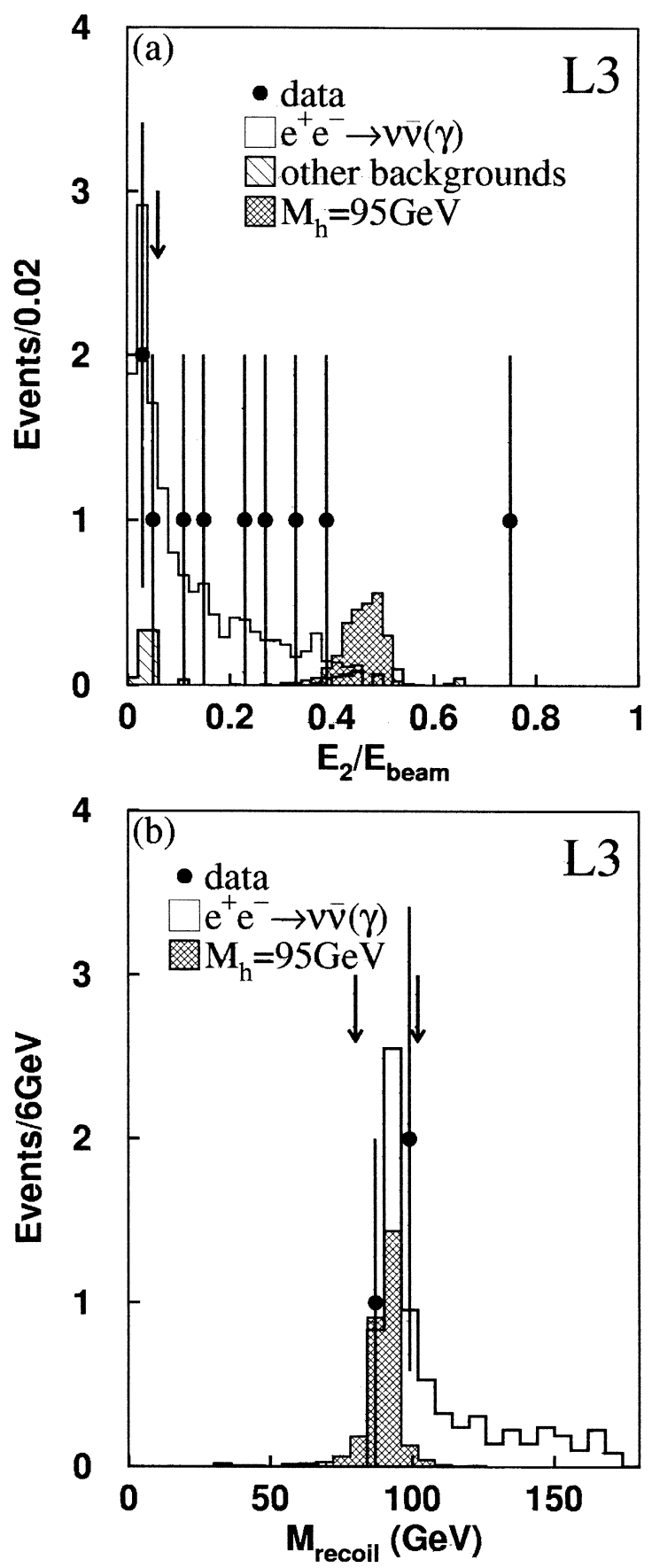

Fig. 2. Distributions for the $\nu \bar{\nu} \gamma \gamma$ final state of (a) the energy of the second most energetic photon normalised to the beam energy and (b) the recoil mass against the two photons in data, background and for a Higgs boson signal with the mass $M_{\mathrm{h}}=$ $95 \mathrm{GeV}$. The signal, corresponding to the Standard Model cross section and $\mathrm{BR}(\mathrm{h} \rightarrow \gamma \gamma)=1$, is superimposed and normalised to the integrated luminosity. 
Table 2

Number of events expected from the Standard Model process $\mathrm{e}^{+} \mathrm{e}^{-} \rightarrow \nu \bar{\nu}(\gamma)$ compared to the observed number of events, for the $\nu \bar{\nu} \gamma \gamma$ final state

\begin{tabular}{ccc}
\hline & Data & $\nu \bar{\nu}(\gamma)$ \\
\hline selection & 3 & 4.3 \\
\hline
\end{tabular}

The energy spectrum of the most energetic photon before any cut is applied on the photon energies is presented in Fig. 1(a). Fig. 1(b) shows the distribution of the recoil mass against the di-photon system after the selection requirements on the photon energies.

We also require the recoil mass against the diphoton system to be consistent with the $\mathrm{Z}$ mass, $\left|M_{\text {recoil }}-M_{\mathrm{Z}}\right|<15 \mathrm{GeV}$. This requirement reduces the background from the $\mathrm{e}^{+} \mathrm{e}^{-} \rightarrow \mathrm{q} \overline{\mathrm{q}}(\gamma)$ process where either a neutral hadron from the $\mathrm{Z}$ decay mimics a photon or a photon in the final state is emitted; in both cases the recoil mass against the photons would be smaller than the $\mathrm{Z}$ mass.

The efficiency of this analysis for selecting $q \bar{q} \gamma \gamma$ events is $43 \%$ for a Higgs boson mass of $95 \mathrm{GeV}$. The number of selected data and background events are presented in Table 1. The dominant background arises from the process $\mathrm{e}^{+} \mathrm{e}^{-} \rightarrow \mathrm{q} \overline{\mathrm{q}}(\gamma)$.

\subsection{The $\nu \bar{\nu} \gamma \gamma$ final state}

The $\nu \bar{\nu} \gamma \gamma$ final state is characterised by the presence of two photons and missing energy in the event. The event selection follows the criteria described in Ref. [23]. To reduce the background from the $\mathrm{e}^{+} \mathrm{e}^{-}$ $\rightarrow \gamma \gamma(\gamma)$ process and from double radiative events with final state particles escaping detection, we require the photon acoplanarity to be greater than $2.5^{\circ}$, the total transverse momentum of the di-photon system to be greater than $3 \mathrm{GeV}$ and the absolute value of the cosine of the polar angle of the missing momentum not to exceed 0.96 .
The energy of the second most energetic photon normalised to the beam energy, $E_{2} / E_{\text {beam }}$, is presented in Fig. 2(a). Fig. 2(b) shows the recoil mass against the two most energetic photons after the selection requirements on the photon energies and the di-photon polar angle.

Furthermore, the recoil mass must be consistent with the $\mathrm{Z}$ boson mass within $\pm 10 \mathrm{GeV}$. The numbers of data and expected background events left after this selection is applied are listed in Table 2. The signal efficiency is $34 \%$ for a Higgs boson of $95 \mathrm{GeV}$ mass. The background is due to the $\mathrm{e}^{+} \mathrm{e}^{-} \rightarrow$ $\nu \bar{\nu}(\gamma)$ process.

\subsection{The $\ell^{+} \ell^{-} \gamma \gamma$ final state}

The $\ell^{+} \ell^{-} \gamma \gamma$ final state is characterised by the presence of two photons and a pair of same type leptons in the event. First low multiplicity events with a photon pair and a lepton pair are preselected.

Electrons are identified as clusters in the BGO with an energy greater than $3 \mathrm{GeV}$ and a matched track. The energy deposited in the hadron calorimeter must be consistent with the tail of an electromagnetic shower. Moreover there must be less than $3 \mathrm{GeV}$ energy deposited in the $\mathrm{BGO}$ in a $10^{\circ}$ cone around the electron direction.

Muons must have a reconstructed track in the muon chambers with a miss distance to the interaction vertex in the $r-\phi$ plane smaller than $300 \mathrm{~mm}$ and a momentum greater than $3 \mathrm{GeV}$. The energy in a $10^{\circ}$ cone around the muon direction must not exceed $3 \mathrm{GeV}$. Also events with one muon and one minimum ionising particle in the calorimeters are accepted. Background events from cosmic rays are removed by requiring at least one hit in the scintillation counters in $\mathrm{a} \pm 5 \mathrm{~ns}$ time window around the beam crossing time.

Taus are identified as jets with one or three tracks in a $10^{\circ}$ cone with an energy greater than $3 \mathrm{GeV}$. The

Table 3

Number of events expected from Standard Model processes compared to the observed number of events, after the preselection and selection steps, for the $\ell^{+} \ell^{-} \gamma \gamma$ final state

\begin{tabular}{lcccccc}
\hline & Data & $\Sigma$ Bkg. & $\mathrm{e}^{+} \mathrm{e}^{-}(\gamma)$ & $\mu^{+} \mu^{-}(\gamma)$ & $\tau^{+} \tau^{-}(\gamma)$ & 4 fermion \\
\hline preselection & 86 & 93.8 & 66.4 & 14.1 & 9.9 & 3.4 \\
selection & 5 & 2.5 & 1.1 & 0.7 & 0.7 & 0.0 \\
\hline
\end{tabular}


energy in the $10^{\circ}-30^{\circ}$ cone must not exceed $30 \%$ of the energy in the $0^{\circ}-10^{\circ}$ cone around the tau direc-
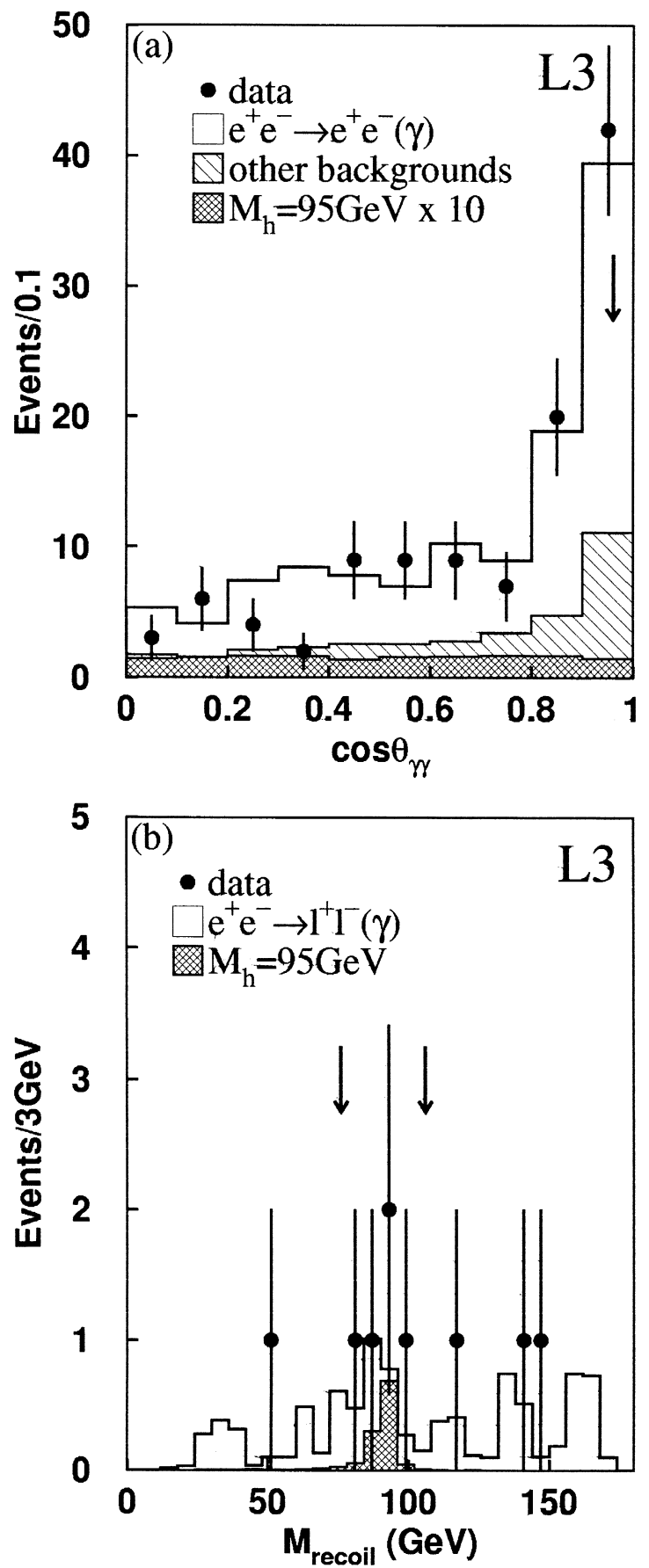

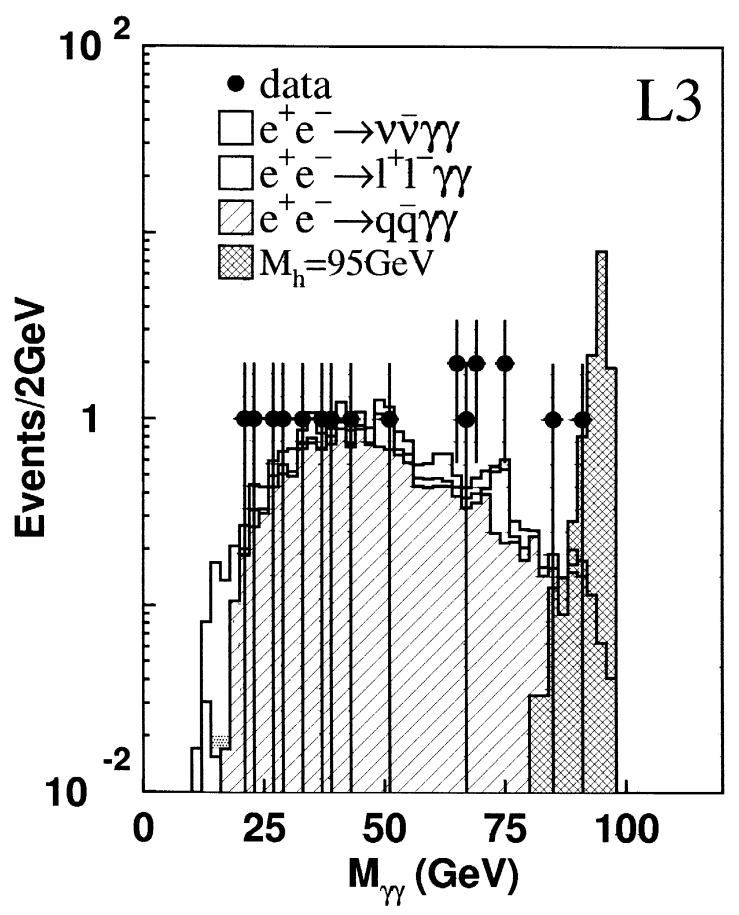

Fig. 4. The distribution of the reconstructed di-photon invariant mass for all $\mathrm{Z}$ final states combined, after the final selection, in data, background and for a Higgs boson signal with the mass $M_{\mathrm{h}}$ $=95 \mathrm{GeV}$. The signal, assuming the Standard Model cross section and $\mathrm{a} \operatorname{BR}(\mathrm{h} \rightarrow \gamma \gamma)=1$, is superimposed and normalised to the integrated luminosity.

tion. In order to maintain a high efficiency, events with only one identified tau are also accepted.

The energy of the most energetic lepton is required to be less than $80 \mathrm{GeV}$ to further reject double radiative di-lepton events. The result of this preselection is reported in Table 3 .

The distribution of the cosine of the polar angle, $\cos \theta_{\gamma \gamma}$, of the di-photon system is shown in Fig. 3(a). Fig. 3(b) shows the recoil mass against the photons after the cuts on the photon energies and on the polar angle of the di-photon system.

Fig. 3. Distributions for the $\ell^{+} \ell^{-} \gamma \gamma$ final state of (a) the cosine of $\theta_{\gamma \gamma}$ for the di-photon system after the preselection and (b) the recoil mass against the two photons in data, background and for a Higgs boson signal with the mass $M_{\mathrm{h}}=95 \mathrm{GeV}$. The signal, corresponding to the Standard Model cross section and a BR( $\mathrm{h} \rightarrow$ $\gamma \gamma)=1$, is superimposed and normalised to the integrated luminosity. A further scale factor of 10 is applied in (a). 


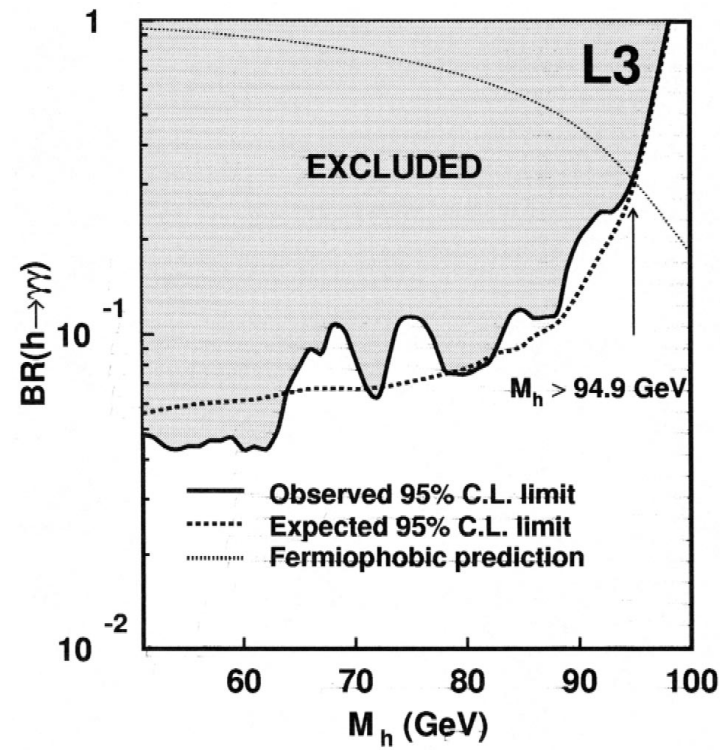

Fig. 5. Excluded values of the $\mathrm{BR}(\mathrm{h} \rightarrow \gamma \gamma)$ as a function of the Higgs mass, in the assumption of a Standard Model production cross section. The expected $95 \%$ confidence level limit and the theoretical prediction are also presented.

In addition, we require the recoil mass to be consistent with the $\mathrm{Z}$ mass, $\left|M_{\text {recoil }}-M_{\mathrm{Z}}\right|<15 \mathrm{GeV}$. The number of events selected in data and expected from background processes is presented in Table 3 . The signal efficiency in the lepton channel is $29 \%$ for a Higgs boson with the mass $M_{\mathrm{h}}=95 \mathrm{GeV}$. The main backgrounds are due to radiative di-lepton events.

\section{Results}

The overall efficiency for selecting $\mathrm{Zh}$ events varies between $36 \%$ and $42 \%$ for Higgs boson masses between $50 \mathrm{GeV}$ and $95 \mathrm{GeV}$, and drops to $30 \%$ at the kinematic limit.

Since no signal is observed in the data, we evaluate the confidence level [24] for the absence of a Higgs signal using the reconstructed di-photon invariant mass as final discriminant variable. This distribution is shown in Fig. 4 for all $\mathrm{Z}$ final states combined.

The calculation of the limits takes into account systematic uncertainties of $1 \%$ from the signal Monte
Carlo statistics, $1.5 \%$ from the simulation of the photon isolation criteria and $4 \%$ on the number of expected background events. The effects from the energy and angular resolution of the photons and the systematic uncertainty on the integrated luminosity are found to be negligible.

Fig. 5 shows the measured upper limits on the $\mathrm{BR}(\mathrm{h} \rightarrow \gamma \gamma)$ as a function of the Higgs mass assuming a Standard Model rate for the $\mathrm{Zh}$ production, along with the expected limits as calculated from a large sample of Monte Carlo experiments. The theoretical prediction is also shown for a fermiophobic Higgs boson as calculated with the HDECAY program [25]. The observed limit for $\mathrm{BR}(\mathrm{h} \rightarrow \gamma \gamma)=1$ is $98 \mathrm{GeV}$. The lower limit on the mass of a fermiophobic Higgs boson is set at

$M_{\mathrm{h}}>94.9 \mathrm{GeV}$ at $95 \%$ confidence level.

The expected mass limit is $95.1 \mathrm{GeV}$.

\section{Acknowledgements}

We wish to express our gratitude to the CERN accelerator division for the excellent performance of the LEP machine. We acknowledge with appreciation the effort of engineers, technicians and support staff who have participated in the construction and maintenance of this experiment.

\section{References}

[1] J. Ellis, M.K. Gaillard, P.V. Nanopoulos, Nucl. Phys. B 106 (1976) 292

[2] K. Hagiwara, R. Szalapski, D. Zeppenfeld, Phys. Lett. B 318 (1993) 155; K. Hagiwara, M.L. Stong, Z. Phys. C 62 (1994) 99; A.G. Akeroyd, Phys. Lett. B 368 (1996) 89; A. Stange, W. Marciano, S. Willenbrock, Phys. Rev. D 49 (1994) 1354; H. Haber, G. Kane, T. Sterling, Nucl. Phys. B 161 (1979) 493; J.F. Gunion, R. Vega, J. Wudka, Phys. Rev. D 42 (1990) 1673.

[3] L. Brücher, R. Santos, Eur. Phys. J. C 12 (2000) 87; L. Brücher, R. Santos, hep-ph/0002027.

[4] M.A. Diaz, T.J. Weiler, hep-ph/9401259.

[5] L3 Collaboration, O. Adriani et al., Phys. Lett. B 292 (1992) 472; L3 Collaboration, M. Acciarri et al., Phys. Lett. B 388 (1996) 409.

[6] OPAL Collaboration, K. Ackerstaff et al., Phys. Lett. B 437 (1998) 218; OPAL Collaboration, G. Abbiendi et al., Phys. Lett. B 464 (1999) 311. 
[7] DELPHI Collaboration, P. Abreu et al., Phys. Lett. B 458 (1999) 431.

[8] L3 Collaboration, B. Adeva et al., Nucl. Instr. and Meth. A 289 (1990) 35; M. Chemarin et al., Nucl. Instr. and Meth. A 349 (1994) 345; M. Acciarri et al., Nucl. Instr. and Meth. A 351 (1994) 300; G. Basti et al., Nucl. Instr. and Meth. A 374 (1996) 293; I.C. Brock et al., Nucl. Instr. and Meth. A 381 (1996) 236; A. Adam et al., Nucl. Instr. and Meth. A 383 (1996) 342.

[9] P. Janot, The HZHA generator, in: G. Altarelli, T. Sjöstrand, F. Zwirner (Eds.), Physics at LEP2, CERN 96-01, 1996, vol. 2, p. 309.

[10] T. Sjöstrand, CERN-TH/7112/93, 1993, revised August 1995; T. Sjöstrand, Comp. Phys. Comm. 82 (1994) 74.

[11] S. Jadach, B.F.L. Ward, Z. Wạs, hep-ph/9912214.

[12] M. Skrzypek et al., Comp. Phys. Comm. 94 (1996) 216; M. Skrzypek et al., Phys. Lett. B 372 (1996) 289.

[13] R. Engel, Z. Phys. C 66 (1995) 203; R. Engel, J. Ranft, S. Roesler, Phys. Rev. D 52 (1995) 1459.
[14] S. Jadach, B.F.L. Ward, Z. Wạs, Comp. Phys. Comm. 79 (1994) 503.

[15] F.A. Berends, R. Kleiss, Nucl. Phys. B 186 (1981) 22.

[16] S. Jadach et al., Phys. Lett. B 390 (1997) 298.

[17] D. Karlen, Nucl. Phys. B 289 (1987) 23.

[18] F.A. Berends, P.H. Daverfeldt, R. Kleiss, Nucl. Phys. B 253 (1985) 441.

[19] F.A. Berends, R. Pittau, R. Kleiss, Comp. Phys. Comm. 85 (1995) 437.

[20] R. Brun et al., Preprint CERN DD/EE/84-1 (Revised 1987).

[21] H. Fesefeldt, RWTH Aachen Report PITHA 85/02, 1985.

[22] S. Bethke et al., Nucl. Phys. B 370 (1992) 310, and references therein.

[23] L3 Collaboration, M. Acciarri et al., Phys. Lett. B 470 (1999) 268.

[24] A. Favara, M. Pieri, hep-ex/9706016.

[25] A. Djouadi, J. Kalinowski, M. Spira, Comp. Phys. Comm. 108 (1998) 56. 